\section{Österreichische Gesellschaft für Verhaltenstherapie (ÖGVT)}

\section{Kongressankündigung \\ "Das Unbewusste in der Verhaltenstherapie" \\ 14. bis 16. Juni 2001 in Bad Aussee}

Donnerstag, 14. Juni 2001

9:00 - 16:30 Uhr:

Workshop: H. Ambühl (Bern) «Die Therapiebeziehung als korrigierende emotionale Erfahrung für den Patienten» 17:30 - 19:00 Uhr:

Festvortrag «30 Jahre ÖGVT»

20:00 Uhr:

Gemeinsames Abendessen mit Posterprämierung

ca. 22:00 Uhr:

Kabarett «Glück gehabt», Dr. Regina Hofer

Freitag, 15. Juni 2001

Vorträge zum Hauptthema:

«Das Unbewusste in der Verhaltenstherapie»

9:15-10:00 Uhr:

P. Fiedler (Heidelberg) «Erinnerung, Vergessen und

Dissoziation: zur Neuropsychologie bewusster und

unbewusster Prozesse»

10:15 - 11:00 Uhr:

D. J. Stein (Stellenbosch, Südafrika)

«Cognition/Unconscious - Studies and Neurobiology»

11:30 - 12:15 Uhr:

F. Resch (Heidelberg) «Affektregulation und Selbstentwicklung: Zur Psychodynamik unbewusster Prozesse» 15:00 - 15:45 Uhr:

H. Ambühl (Bern) «Die Therapeut-Patient-Beziehung unter dem Gesichtspunkt der Konfliktdynamik»

16:00 - 16:45 Uhr:

Tanja Michael (Oxford) «Implizites Gedächtnis bei der posttraumatischen Belastungsstörung»

17:15 - 18:00 Uhr:

J. Margraf (Basel) «Unbewusste Prozesse, psychische Störungen und moderne Klinische Psychologie»

20:00 Uhr:

Gesellschaftsabend
Samstag, 16. Juni 2001

Ganztägige Workshops

P. Fiedler (Heidelberg)

«Dissoziative Identitätsstöungen und multiple Persönlichkeit:

Konzepte, Filmbeispiel und Diskussion»

R. F. W. Diekstra (Holland)

«Traumarbeit und kognitive Verhaltenstherapie» (angefragt)

Information und Programm:

ÖGVT, Österreichische Gesellschaft für Verhaltenstherapie A-1090 Wien, Kolingasse 11/9

Tel. ++43-1-3197022, Fax ++43-1-3197240

E-mail oegvt@xpoint.at,http://www.user.xpoint.at/oegvt

\section{Institut für Fort- und Weiterbildung in Klinischer Verhaltenstherapie, Bad Dürkheim (IFKV)}

Das Institut für Fort- und Weiterbildung in klinischer Verhaltenstherapie e.V. (staatlich anerkanntes Ausbildungsinstitut) Bad Dürkheim bietet wiederum ab September 2001 eine Weiterbildung zum/zur Supervisor/in mit Schwerpunkt Verhaltenstherapie an.

Theorie:

- Allgemeine versus verhaltensttherapeutische Supervision

- Techniken der Supervision

- Supervisionsmodelle verschiedener Therapieschulen

- Organisations- und Teamsupervision

- Entwicklungsprozesse in der Supervision

Praxis:

- Praxisseminare

- Supervision der Supervision durch Lehrtherapeuten

- Co-Supervision und Intervision

Anmeldung zum Info-Gespräch am 14.03.2001 und weitere Auskünfte:

IFKV-Sekretariat, Kurbrunnenstr. 21 a, 67098 Bad Dürkheim Tel. 06322/68019, Fax 06322/68010, E-mail info@ifkv.de Internet: http://www.ifkv.de 